\title{
The Metal-Insulator Transition of $\mathrm{NbO}_{2}$ : an Embedded Peierls Instability
}

\author{
V. Eyert周 \\ Institut für Physik, Universität Augsburg, 86135 Augsburg, Germany
}

(Dated: May 28, 2018)

\begin{abstract}
Results of first principles augmented spherical wave electronic structure calculations for niobium dioxide are presented. Both metallic rutile and insulating low-temperature $\mathrm{NbO}_{2}$, which crystallizes in a distorted rutile structure, are correctly described within density functional theory and the local density approximation. Metallic conductivity is carried to equal amounts by metal $t_{2 g}$ orbitals, which fall into the one-dimensional $d_{\|}$band and the isotropically dispersing $e_{g}^{\pi}$ bands. Hybridization of both types of bands is almost negligible outside narrow rods along the line $\mathrm{X}-\mathrm{R}$. In the lowtemperature phase splitting of the $d_{\|}$band due to metal-metal dimerization as well as upshift of the $e_{g}^{\pi}$ bands due to increased $p$ - $d$ overlap remove the Fermi surface and open an optical band gap of about $0.1 \mathrm{eV}$. The metal-insulator transition arises as a Peierls instability of the $d_{\|}$band in an embedding background of $e_{g}^{\pi}$ electrons. This basic mechanism should also apply to $\mathrm{VO}_{2}$, where, however, electronic correlations are expected to play a greater role due to stronger localization of the $3 d$ electrons.
\end{abstract}

PACS numbers: 71.20.-b, 71.30.+h, 72.15.Nj

Despite intense work over decades the metal-insulator transition (MIT) of $\mathrm{VO}_{2}$ has remained a matter of controversy [1, 2, 3, 4, 5, 6, 7]. This is related to the simultaneous occurrence of a structural transformation from the high-temperature rutile phase to a distorted monoclinic structure, which is characterized by (i) pairing of the metal atoms within chains parallel to the rutile $c$ axis and (ii) their lateral zigzag-like displacement [8]. Electronic states near the Fermi energy are of mainly $\mathrm{V} 3 d$ $t_{2 g}$ character. They separate into the $d_{\|}$band, which mediates $\mathrm{V}-\mathrm{V}$ overlap along the metal chains, and the remaining $e_{g}^{\pi}$ bands [3]. At the transition, splitting of the $d_{\|}$band and upshift of the $e_{g}^{\pi}$ bands due to increased metal-oxygen overlap produce a finite band gap. Dispute centers about the question, whether the $d_{\|}$splitting is caused by metal dimerization or by increased electronic correlations resulting from the reduced screening by the $e_{g}^{\pi}$ electrons [3, 44. State of the art band calculations gave strong hints of a structural instability but were not able to reproduce the insulating gap due to the shortcomings of the local density approximation (LDA) [5].

Interestingly, only very few studies have dealt with the neighbouring dioxides, which display related phenomena. $\mathrm{NbO}_{2}$ likewise undergoes a MIT (at $1081 \mathrm{~K}$ ) and a simultaneous structural transition from rutile to a distorted variant having a body-centered tetragonal (bct) lattice [9, 10]. Despite the differences in long range order, local deviations from rutile are the same as in $\mathrm{VO}_{2}$, i.e. niobium atoms dimerize and experience lateral displacements at the transition [11, 12, 13]. In contrast, the metallic oxides $\mathrm{MoO}_{2}, \mathrm{WO}_{2}, \mathrm{TcO}_{2}$, and $\alpha-\mathrm{ReO}_{2}$ all crystallize in the same monoclinic structure as $\mathrm{VO}_{2}$ 14. No doubt the phase transitions of $\mathrm{VO}_{2}$ and $\mathrm{NbO}_{2}$ as well as the destabilization of the rutile structure in all these dioxides call for a unified description [2, 3, 10. Yet, although work on the dioxides neighbouring $\mathrm{VO}_{2}$ would help clarifying the nature of the MIT, there are only few theoretical investigations in this direction 115, 16, 17. For this reason, a complete and widely accepted picture of the rutile-related transition metal dioxides has not yet evolved.

Here we present first principles electronic structure calculations for $\mathrm{NbO}_{2}$. These are the first calculations at all for the insulating phase and the first calculations for rutile $\mathrm{NbO}_{2}$, which used the experimental crystal structure. Both phases are correctly described within density functional theory (DFT) and the LDA. The results strongly support the band theoretical point of view of the MIT as arising predominantly from a Peierls-type instability of the rutile phase.

Evidence for the MIT being driven by strong metalmetal bonding was first inferred from the small and almost temperature independent paramagnetic susceptibility below the transition temperature as well as from the enhanced thermal expansion of the $c$ lattice parameter just above $T_{c}$ 18, 19, 20, 21]. Additional support came from strong decrease of $T_{c}$, nonlinear variation of $c$, and crossover of the magnetic susceptibility to Curie-Weiss behaviour on substitution of $\mathrm{Ti}$ for $\mathrm{Nb}$, which destroys the metal-metal bonding [19, 20, 21].

According to neutron-diffraction experiments the bct structure of insulating $\mathrm{NbO}_{2}$ has 16 formula units per cell, space group $I 4_{1} / a\left(C_{4 h}^{6}\right)$, and lattice constants $a \approx 2 \sqrt{2} a_{R}$ and $c \approx 2 c_{R}$ [11, 12, 13, where $a_{R}=$ $4.8464 \AA$ and $c_{R}=3.0316 \AA$ are the rutile lattice parameters 22]. The two different $\mathrm{Nb}-\mathrm{Nb}$ distances of 2.7 and $3.3 \AA$ as arising from the metal-metal pairing along the rutile $c$ axis [13] deviate considerably from the rutile value $c_{R}$. In addition, the zigzag-like in-plane displacement of niobium atoms parallel to either the [110] or [110] direction of the rutile subcell leads to a variation of $\mathrm{Nb}-\mathrm{O}$ distances between 1.91 and $2.25 \AA$, which deviate from the high-temperature values of 2.00 and $2.08 \AA$ 12, 22]. Critical scattering observed above $T_{c}$ at the 
tetragonal wave vector $\mathbf{q}_{P}=(1 / 4,1 / 4,1 / 2)$ pointed to a soft phonon mode, which, however, could not be clearly identified [13, 23, 24]. Yet, using a shell model, Gervais and Kress were able to reveal softening of a $P$ point phonon with a displacement pattern consistent with the low-temperature structure 25].

Room-temperature UPS and XPS experiments revealed a $9 \mathrm{eV}$ wide occupied group of bands, which falls into the $1 \mathrm{eV}$ wide $\mathrm{Nb} 4 d$ bands just below $E_{F}$ and a $6 \mathrm{eV}$ wide group of $\mathrm{O} 2 p$ bands at higher binding energies [26]. The room temperature optical band gap was estimated to about $0.5 \mathrm{eV}$ [2]. By and large, photoemission data agree with band structure and $\mathrm{X} \alpha$ cluster calculations [15, 16, 27, 28], which, due to the complexitiy of the low-temperature structure, only addressed to the rutile phase. Only Sasaki et al. accounted for the bct structure by studying $\mathrm{Nb}_{2} \mathrm{O}_{10}$ clusters with either short or long $\mathrm{Nb}-\mathrm{Nb}$ distance and deduced a band gap of $0.68 \mathrm{eV}$; however, oxygen positions were not relaxed and the same value for all Nb-O distances used [16]. Finally, calculated Fermi surfaces and generalized susceptibilities agreed with experiment in that they could not find a soft-mode instability [15, 28].

The present calculations were performed using the scalar-relativistic augmented spherical wave (ASW) method [29]. Both the LDA and the generalized gradient approximation were applied with essentially no differences in the electronic structure. Crystallographic data as given by Bolzan et al. [22] and Pynn et al. [13] were used. In order to account for the loose packing of the crystal structures empty spheres, i.e. pseudo atoms without a nucleus, were included to model the correct shape of the crystal potential in large voids. Optimal empty sphere positions and radii of all spheres were automatically determined [30]. As a result, three and 14 inequivalent empty sphere types with radii ranging from 0.96 to $2.49 a_{B}$ were included in the rutile and bct cell, respectively, keeping the linear overlap of niobium and oxygen spheres below $18.5 \%$. The basis set comprised $\mathrm{Nb} 5 s, 5 p, 4 d$ and $\mathrm{O} 2 s, 2 p$ as well as empty sphere states. Brillouin zone sampling was done using an increased number of $\mathbf{k}$ points ranging from 18 to 1800 and 12 to 552 points within the respective irreducible wedge.

Partial densities of states (DOS) resulting from calculations for the rutile and bct structures are displayed in Fig. I. The gross features are similar in both phases. Three groups of bands are identified. While bands in the energy range from $\approx-9$ to $-3 \mathrm{eV}$ are dominated by O $2 p$ states, the two higher lying groups at and above the Fermi energy derive mainly from $\mathrm{Nb} 4 d$ orbitals. All other states play only a negligible role in the energy interval shown. Crystal field splitting causes nearly complete energetical separation of the $\mathrm{Nb} 4 d$ bands into two groups of $t_{2 g}$ and $e_{g}^{\sigma}$ symmetry. Contributions of the O $2 p$ and $\mathrm{Nb} 4 d$ states to the upper two and the lower group, respectively, are indicative of covalent bonding.
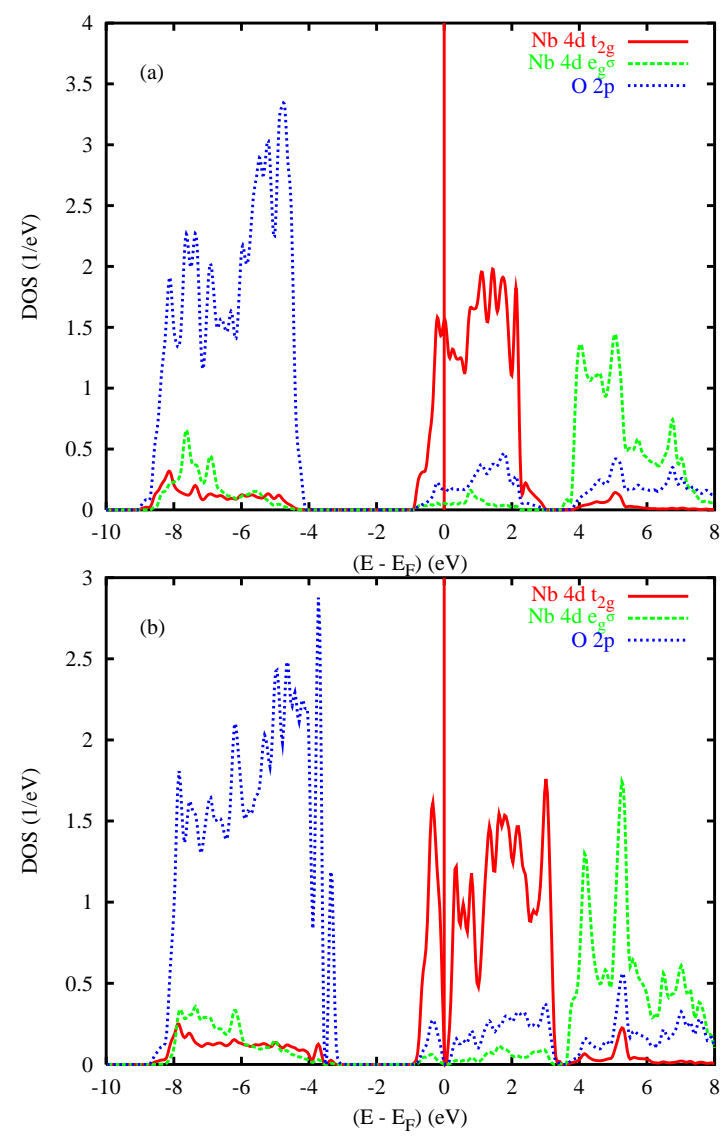

FIG. 1: Partial DOS of (a) metallic and (b) insulating $\mathrm{NbO}_{2}$ (per f.u.). Slight broadening is due to the DOS calculation scheme 31.

Our results for insulating and metallic $\mathrm{NbO}_{2}$ are in good agreement with the photoemission experiments 26] and the previous calculations [15, 16, 27, 28]. Differences with the latter could be traced back to the fact that these calculations were not self-consistent or, due to lack of crystallographic data, used a rutile structure obtained from symmetrizing the bct structure.

The results for both phases differ with respect to the energetical position of the O $2 p$ group of bands as well as the shape of the $t_{2 g}$ partial DOS. In Fig. 2 these DOS are further separated into their symmetry components. To this end orbitals were transformed to a local coordinate system with the $z$ and $x$ axis parallel to the apical axis of the local octahedron and the rutile $c$ axis, respectively [17. While the $d_{x^{2}-y^{2}}\left(\equiv d_{\|}\right)$and $d_{y z}$ states mediate $\sigma$-type $d$ - $d$ overlap along the rutile $c$ and $a$ axes, respectively, the $d_{x z}$ states account for $\pi$-type overlap across the chains. Note that the latter two orbitals combine into the $e_{g}^{\pi}$ states. The $t_{2 g}$ groups of bands are displayed in Fig. 3, where the $d_{x^{2}-y^{2}}$ bands are highlighted by bars with widths proportional to the $d_{x^{2}-y^{2}}$-contribution to the respective wave function. Thus, states with vanishing bars are of pure $e_{g}^{\pi}$ character (apart from small $e_{g}^{\sigma}$ as 

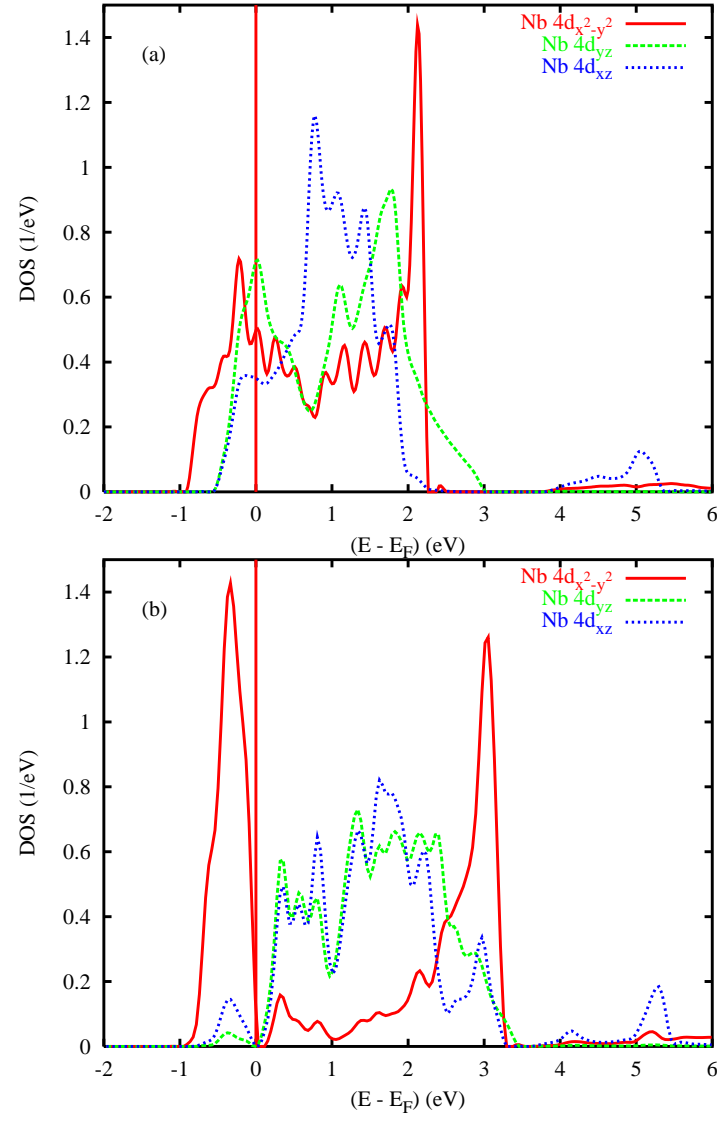

FIG. 2: Partial $t_{2 g}$ DOS of (a) metallic and (b) insulating $\mathrm{NbO}_{2}$ (per f.u.).

well as $\mathrm{O} 2 p$ contributions).

Most striking in Fig. 3(a) is the nearly perfect onedimensional dispersion of the $d_{x^{2}-y^{2}}$ bands parallel to the rutile $c$ axis, i.e. parallel to the niobium chains, which gives rise to the pronounced double peak structure in the partial DOS, Fig. 2(a). In contrast, dispersion of the $e_{g}^{\pi}$ bands is of the same order in all directions. This leads to the single broad structure of the $d_{x z}$ partial DOS, while the $d_{y z}$ states fall into two maxima due to the aforementioned inplane $d$ - $d$ overlap. The DOS at $E_{F}$ is comprised from similar contributions from all three $t_{2 g}$ orbitals explaining the rather isotropic electrical conductivity. Note that, except for the bands along the line $\mathrm{X}-\mathrm{R}$, the $d_{\|}$ states hardly hybridize with the $e_{g}^{\pi}$ states and thus coupling between both types of bands is mainly via charge conservation. For this reason, the $d_{x^{2}-y^{2}}$ band may be regarded as a one-dimensional band in a three-dimensional embedding background of $e_{g}^{\pi}$ bands.

On going to insulating $\mathrm{NbO}_{2}$ two distinct changes occur. i) The previously one-dimensional $d_{x^{2}-y^{2}}$ band is split into bonding and antibonding branches due to Nb$\mathrm{Nb}$ dimerization within the chains. As a consequence, the separation of the two peaks in the $d_{x^{2}-y^{2}}$ partial DOS has increased by more than $1 \mathrm{eV}$ and the contributions
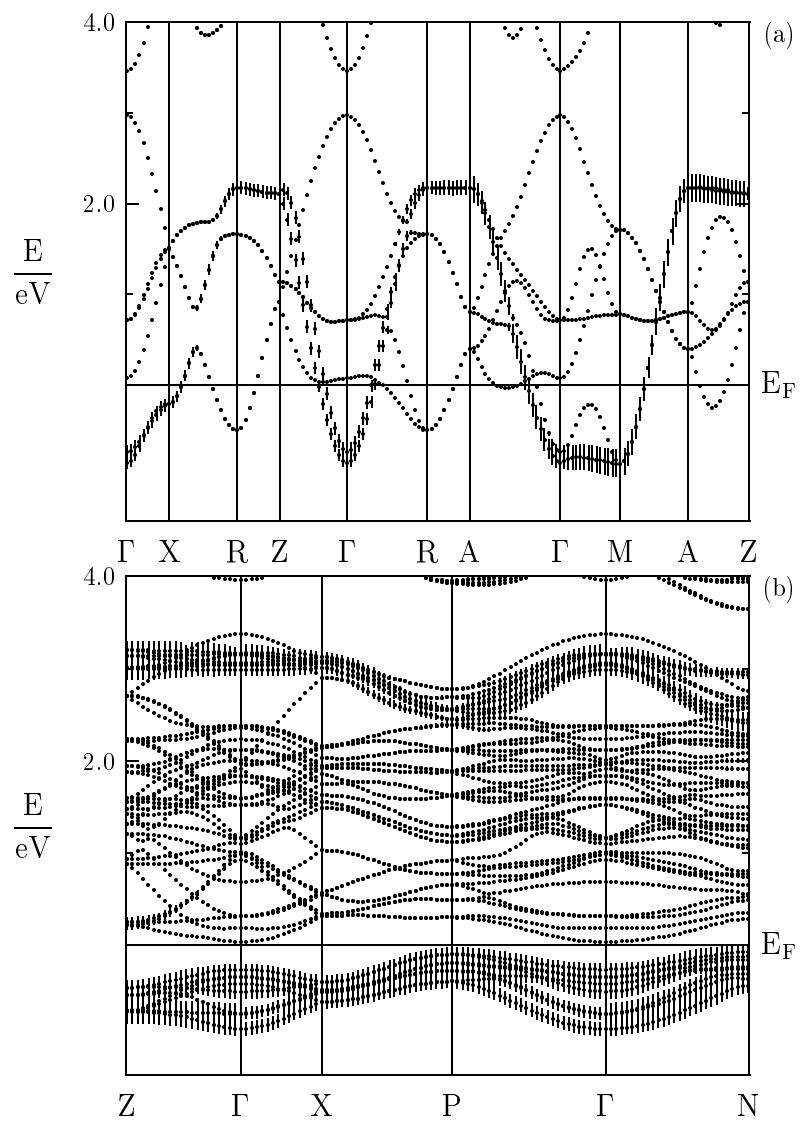

FIG. 3: Weighted band structure of (a) metallic and (b) insulating $\mathrm{NbO}_{2}$ (see text).

in between have been considerably reduced. ii) The $e_{g}^{\pi}$ states experienced energetical upshift by $\approx 0.5 \mathrm{eV}$ due to increased $p$ - $d$ overlap, causing almost complete depopulation of these orbitals. This is due to the much reduced $\mathrm{Nb}-\mathrm{O}$ distances arising from the zigzag-like displacement of the $\mathrm{Nb}$ atoms. However, the lateral displacements also affect the inplane metal-metal bonding. For this reason, the two peak structure of the $d_{y z}$ partial DOS has vanished and both $e_{g}^{\pi}$ partial DOS have become more similar. Taken together these changes lead to complete separation of the low lying $d_{x^{2}-y^{2}}$ bands from the $e_{g}^{\pi}$ states, which gives rise to a band gap of $0.1 \mathrm{eV}$. This value was determined from checking band extrema on a $16 \times 16 \times 16$ mesh within the Brillouin zone. Note that the hybridization between both types of bands is still negligible.

To conclude, the electronic structures of both metallic and insulating $\mathrm{NbO}_{2}$ are well described within DFT and LDA. The near $E_{F}$ electronic structure of metallic $\mathrm{NbO}_{2}$ consists of two very weakly hybridizing types of $t_{2 g}$ bands, namely, $d_{\|}$states with one-dimensional dispersion parallel to the $\mathrm{Nb}$ chains and isotropically dispersing $e_{g}^{\pi}$ bands. In the low-temperature structure $\mathrm{Nb}-\mathrm{Nb}$ dimerization splits the $d_{\|}$band into bonding and antibonding branches, whereas the $e_{g}^{\pi}$ states shift to higher energies 
due to reduced Nb-O distances. Since coupling between both types of bands is still by charge conservation rather than hybridization, the insulating state is interpreted as due to a Peierls instability of the $d_{\|}$bands in an embedding reservoir of $e_{g}^{\pi}$ electrons.

The results complement previous work on $\mathrm{VO}_{2}$ as well as $\mathrm{MoO}_{2}$ [5, 17] and give rise to a unified picture for the early transition metal dioxides, which explains (i) destabilization of the rutile structure and (ii) the metalinsulator transition of the $d^{1}$ members. Since the $4 d$ bands are broader than the $3 d$ bands metal-metal bonding is stronger in the fourth row dioxides this leading to the higher transition temperature of $\mathrm{NbO}_{2}$ as compared to $\mathrm{VO}_{2}$. In contrast, the absence of a gap in LDA calculations for the latter compound might be indicative of a considerable amount of electronic correlations. Here, a combination of LDA and dynamical mean field theory as recently successfully applied to $\mathrm{V}_{2} \mathrm{O}_{3} 32$ might be more appropriate.

Valuable discussions with U. Eckern, K.-H. Höck, S. Horn, P. S. Riseborough, and D. Vollhardt are gratefully acknowledged. This work was supported by the Deutsche Forschungsgemeinschaft through Sonderforschungsbereich 484 .

* http://www.physik.uni-augsburg.de/ eyert/

[1] J. B. Goodenough, Phys. Rev. 117, 1442 (1960).

[2] D. Adler, Rev. Mod. Phys. 40, 714 (1968).

[3] J. B. Goodenough, in: Progress in Solid State Chemistry, edited by H. Reiss (Pergamon Press, Oxford, 1971), Vol. 5, p. 145.

[4] A. Zylbersztejn and N. F. Mott, Phys. Rev. B 11, 4383 (1975).

[5] R. M. Wentzcovitch, W. W. Schulz, and P. B. Allen, Phys. Rev. Lett. 72, 3389 (1994); 73, 3043 (1994).

[6] T. M. Rice, H. Launois, and J. P. Pouget, Phys. Rev. Lett. 73, 3042 (1994).

[7] M. Imada, A. Fujimori, and Y. Tokura, Rev. Mod. Phys. 70, 1039 (1998).

[8] G. Andersson, Acta Chim. Scand. 10, 623 (1956); J. M. Longo and P. Kierkegaard, Acta Chim. Scand. 24, 420
(1970).

[9] R. F. Janninck and D. H. Whitmore, J. Phys. Chem. Solids 27, 1183 (1966).

[10] T. Sakata, K. Sakata, and I. Nishida, phys. stat. sol. 20, K155 (1967).

[11] B.-O. Marinder, Ark. Kemi 19, 435 (1962).

[12] A. K. Cheetham and C. N. R. Rao, Acta Cryst. B 32, 1579 (1976).

[13] R. Pynn, J. D. Axe, and R. Thomas, Phys. Rev. B 13, 2965 (1976).

[14] D. B. Rogers, R. D. Shannon, A. W. Sleight, and J. L. Gillson, Inorg. Chem. 8, 841 (1969); L. F. Mattheiss, Phys. Rev. B 13, 2433 (1976).

[15] M. Posternak, A. J. Freeman, and D. E. Ellis, Phys. Rev. B 19, 6555 (1979).

[16] T. A. Sasaki and T. Soga, Physica B 111, 304 (1981).

[17] V. Eyert, Habilitation thesis, Universität Augsburg, 1998; V. Eyert, R. Horny, K.-H. Höck, and S. Horn, J. Phys.: Cond. Matt. 12, 4923 (2000).

[18] G. Brauer, Z. Anorg. Allg. Chem. 248, 1 (1941).

[19] W. Rüdorff and H.-H. Luginsland, Z. Anorg. Allg. Chem. 334, 125 (1964).

[20] K. Sakata, J. Phys. Soc. Japan 26, 582 (1969); 26, 867 (1969); 26, 1067 (1969).

[21] K. Sakata, I. Nishida, M. Matsushima, and T. Sakata, J. Phys. Soc. Japan 27, 506 (1969).

[22] A. A. Bolzan, C. Fong, B. J. Kennedy, and C. J. Howard, J. Solid State Chem. 113, 9 (1994); Acta Cryst. B 53, 373 (1997).

[23] S. M. Shapiro, J. D. Axe, G. Shirane, and P. M. Raccah, Solid State Commun. 15, 377 (1974).

[24] R. Pynn, J. D. Axe, and P. M. Raccah, Phys. Rev. B 17, 2196 (1978).

[25] F. Gervais and W. Kress, Phys. Rev. B 31, 4809 (1985).

[26] N. Beatham and A. F. Orchard, J. El. Spec. Rel. Phen. 16, 77 (1979).

[27] C. Umrigar and D. E. Ellis, Phys. Rev. B 21, 852 (1980).

[28] J. H. Xu, T. Jarlborg, and A. J. Freeman, Phys. Rev. B 40, 7939 (1989).

[29] A. R. Williams, J. Kübler, and C. D. Gelatt, Jr., Phys. Rev. B 19, 6094 (1979); V. Eyert, Int. J. Quantum Chem. 77, 1007 (2000).

[30] V. Eyert and K.-H. Höck, Phys. Rev. B 57, 12727 (1998).

[31] M. S. Methfessel and A. T. Paxton, Phys. Rev. B 40, 3616 (1989).

[32] K. Held, G. Keller, V. Eyert, D. Vollhardt, and V. Anisimov, Phys. Rev. Lett. 86, 5345 (2001). 